\title{
Long-term hemodialysis may affect enlarged perivascular spaces in maintenance hemodialysis patients: evidence from a pilot MRI study
}

\author{
Hao Wang ${ }^{1}$, Xue Han ${ }^{2}$, Mingan $\mathrm{Li}^{1}$, Zheng-Han Yang ${ }^{1}$, Wen-Hu Liu ${ }^{2}$, Zhen-Chang Wang ${ }^{1}$ \\ ${ }^{1}$ Department of Radiology, Beijing Friendship Hospital, Capital Medical University, Beijing, China; ${ }^{2}$ Department of Nephrology, Faculty of Kidney \\ Diseases, Beijing Friendship Hospital, Capital Medical University, Beijing, China
}

Contributions: (I) Conception and design: H Wang, ZC Wang; (II) Administrative support: ZC Wang, WH Liu, ZH Yang; (III) Provision of study materials or patients: X Han, WH Liu; (IV) Collection and assembly of data: H Wang, X Han; (V) Data analysis and interpretation: H Wang; (VI) Manuscript writing: All authors; (VII) Final approval of manuscript: All authors.

Correspondence to: Zhen-Chang Wang. Department of Radiology, Beijing Friendship Hospital, Capital Medical University, No. 95 Yong An Road, Xicheng District, Beijing, 100050, China. Email: cjr.wzhch@vip.163.com.

Background: Hemodialysis (HD) causes various nervous system abnormalities. Alterations in white matter (WM) microstructure after long-term HD have been reported in a few previous studies; however, no studies have been performed to investigate enlarged perivascular spaces (PVS) in WM regions. We measured cerebral blood flow (CBF) and white matter volume (WMV) in HD patients to assess enlarged PVS severity in the WM across the whole brain and suggest possible explanations for this.

Methods: Fifty-one HD patients and 51 age-, sex-, and education-matched healthy controls (HCs) were recruited. The number of enlarged PVS in the centrum semiovale (CS), cerebral watershed (CW), and basal ganglia (BG) regions were assessed by T2-weighted MRI. CBF was estimated by arterial spin labeling (ASL), which is a non-invasive perfusion imaging technique. WMV was assessed by the computational anatomy toolbox (CAT12), which is a statistical analysis package. Differences in descriptive variables (two-tailed $t$-tests, $\chi^{2}$ tests, Mann-Whitney $U$ tests, and Friedman $M$ tests), an intra-class correlation between radiologists, the relationship between enlarged PVS number and HD duration, normalized CBF and WMV (multiple regression), and group differences in CBF and WMV \{voxel-wise $t$-tests with age and sex as covariates [cluster size $>50$ voxels, false discovery rate $($ FDR) corrected, $\mathrm{P}<0.05]\}$ were assessed.

Results: HD patients displayed a more significant number of CS-PVS and CW-PVS in WM regions compared with the HCs, but there was no significant difference in the number of BG-PVS. The number of CS-PVS and CW-PVS were positively associated with HD duration. The number of CW-PVS was positively associated with CBF changes and WMV alteration in HD patients. Meanwhile, significant differences in the blood pressure (BP) readings pre-HD, intra-HD, and post-HD were observed in HD patients. Compared with the HCs, the HD patients showed higher CBF in the CS, CW, and BG regions $(\mathrm{P}<0.05)$. Hence, decreased WMV in the CS, CW, and BG regions were shown in the HD patients compared with the $\mathrm{HCs}(\mathrm{P}<0.05)$.

Conclusions: Enlarged CS-PVS and CW-PVS on MRI might be a feature of long-term HD patients. Enlarged CW-PVS number is associated with higher CBF in the CW region and lower WMV in the CW region in $\mathrm{HD}$ patients.

Keywords: Hemodialysis; perivascular spaces; voxel-based morphometry; arterial spin labeling

Submitted Nov 07, 2020. Accepted for publication Jun 23, 2021.

doi: 10.21037/qims-20-1246

View this article at: https://dx.doi.org/10.21037/qims-20-1246 


\section{Introduction}

End-stage renal disease (ESRD) is a result of chronic renal dysfunction (1), which occurs when chronic kidney disease (CKD) progresses to a glomerular filtration rate (GFR) of $<15 \mathrm{~mL} / \mathrm{min} / 1.73 \mathrm{~m}^{2}$. In recent years, ESRD has become an increasing global health problem (2). To clear away surplus urea and other metabolic waste from the body, patients with ESRD generally undergo regular HD three times per week. However, ESRD patients may experience neurologic complications such as dialysis disequilibrium syndrome, hypertensive encephalopathy, osmotic myelinolysis, intracranial hemorrhage, stroke, and dementia (3-5), which may be related to ESRD itself or HD. These nervous system abnormalities are probably associated with brain edema or tissue swelling, which may be caused by the osmotic gradient between brain tissue and plasma during HD (6).

HD may gradually cause WM microstructure modifications (7). In a previous study, researchers explored the effects of long-term HD on WM integrity using the diffusion tensor imaging (DTI) technique by comparing fractional anisotropy (FA) values between ESRD patients and normal subjects (8). The research showed that FA values in many WM regions were significantly decreased and that reductions in FA values were negatively correlated with the duration of HD. Zhang et al. showed that abnormalities in associative and radiation fiber tracts of ESRD patients resulting in brain edema were associated with cognitive function decrease (9). Yi et al. found that widespread impairments in corona radiata and thalamic radiations may have influenced cognitive function in ESRD patients, and serum urea levels were related to these WM changes (10). The existing literature has focused on exploring alterations in WM microstructure, but few studies have investigated visible WM alterations, such as enlarged PVS in WM regions (11-13).

Enlarged PVS, also known as Virchow-Robin spaces, are fluid-filled cavities surrounding the penetrating vessels, which hold metabolic waste cleared from the brain, and are a conduit for fluid transport (14). Enlarged PVS are visible in T1-weighted and T2-weighted MRI images as linear, ovoid, or round cerebrospinal fluid (CSF) intensities in the centrum semiovale (CS) and basal ganglia (BG) (15). Although it is common to see many enlarged PVS at different ages, enlarged PVS have been documented in elevated numbers in the elderly population (16). Previous studies have reported that enlarged PVS are related to impaired cognitive function (17), small vessel disease (SVD) (18), and inflammatory aggravations in multiple sclerosis (19).

ASL imaging has been used as a non-invasive perfusion imaging technique to investigate brain perfusion alterations in HD patients $(20,21)$. Meanwhile, previous studies showed that voxel-based morphometry could be utilized to exhibit cerebral gray matter (GM) morphological changes in HD patients $(22,23)$. The existing studies have focused on exploring the GM changes in ESRD patients (24). There have been no studies investigating WM changes, and correlations between WM alteration levels and enlarged PVS, in HD patients.

The purpose of this study was to explore the extent of enlarged PVS in WM regions of the brain in maintenance HD patients, using T2-weighted MRI. In addition, we investigated whether enlarged PVS in the CS, CW, and BG regions share the same pathogenesis. Correlation analyses were used to explore the associations of HD duration and biochemical variables with enlarged PVS. To explore the underlying mechanisms of enlarged PVS, ASL and CAT12 techniques were performed in ESRD patients. To our knowledge, this is the first study to investigate enlarged PVS alterations in WM regions in maintenance HD patients.

\section{Methods}

\section{Participants}

Fifty-one HD patients (30 male and 21 female) were recruited. The underlying reason for requiring HD in these patients was primary hypertensive nephropathy or glomerulonephritis. Fifty-one normal age- and sex-matched subjects (32 male and 19 female) were enrolled as HCs. GFR was used to diagnose all HD patients. When the GFR $<15 \mathrm{~mL} / \mathrm{min} / 1.73 \mathrm{~m}^{2}$, patients need to be hospitalized for maintenance $\mathrm{HD}$, and the duration of dialysis is longer than six months. All HD patients underwent HD treatments three times per week as a standard, and a single HD session was approximately $4 \mathrm{~h}$. To aviod for the acute effects of HD treatment, all HD patients were scanned one day after the last HD session each week. The exclusion criteria for participation in the study were (I) diabetic nephropathy; (II) other dialysis therapy; (III) brain disease history, including stroke, cerebral trauma, cerebral infarct, and tumors; (IV) psychiatric disorder history; (V) vertigo or intolerance of MR scanning; and (VI) history of drug or alcohol abuse. The study was conducted following the Declaration of 
Helsinki (as revised in 2013). This study was approved by the Medical Ethics Committee of Beijing Friendship Hospital, Capital Medical University. Informed consent was obtained from each participant included in the study.

\section{MRI data acquisition}

All subjects underwent imaging performed using a 3.0-Tesla MR system with an eight-channel phased array coil (Discovery MR750W, General Electric, Milwaukee, Wisconsin, USA). All participants were required to refrain from intake caffeine-containing foods or drinks before MRI scanning. Soft but comfortable, foam padding was employed to minimize head motion, and earplugs were used to reduce scanner noise. The perfusion imaging used was pseudo-continuous arterial spin labeling (PCASL) sequence (25). The major parameters for PCASL imaging using fast spin echo (FSE) imaging readout were: 36 axial slices, slice thickness $=4 \mathrm{~mm}$; labeling duration/post label delay $=2,025 \mathrm{~ms} / 2,025 \mathrm{~ms}$; repetition time/echo time (TR/ TE) $=4,844 / 10.5 \mathrm{~ms}$; matrix $=128 \times 128$; field of view (FOV) $=240 \times 240 \mathrm{~mm}$ across whole-brain coverage; number of excitations $=3$; spiral-in readout of 512 sample points with eight arms; and number of label and control images $=72$. The overall acquisition time of the resting-state ASL performance was 4 min $41 \mathrm{~s}$. A T1-weighted structural image was acquired using the $3 \mathrm{D}$ brain volume imaging (BRAVO) sequence [TR $=8.8 \mathrm{~ms}$, $\mathrm{TE}=3.5 \mathrm{~ms}$; inversion time $(\mathrm{TI})=450 \mathrm{~ms} ; 196$ slices with 1 - $\mathrm{mm}$ thickness (no gap); matrix $=256 \times 256$; FOV $=24 \mathrm{~cm} \times 24 \mathrm{~cm}$; flip angle $\left.=15^{\circ}\right]$. A $\mathrm{T} 2$-weighted image was generated using the FSE sequence ( $\mathrm{TR}=7,299 \mathrm{~ms}, \mathrm{TE}=169 \mathrm{~ms} ; 20$ slices with 6-mm thickness; matrix $=256 \times 256 ; \mathrm{FOV}=24 \mathrm{~cm} \times 24 \mathrm{~cm}$; flip angle $=15^{\circ}$ ). During the scans, all subjects were asked to relax, keep their eyes closed, think of nothing in particular, and avoid falling asleep.

\section{Enlarged PVS assessment}

Enlarged PVS were defined as fluid-filled entities presenting as sharply delineated ovoid or round structures, depending on the imaging plane (26). Enlarged PVS is located in the CS, BG, and CW, with CSF intensity signal in T2WI, size $<3 \mathrm{~mm}$, and follow the typical course of a vessel. Enlarged PVS were counted in the plane with the largest number visible (Figure 1). The definition of CS, BG, and CW reference the study by Potter et al. (27). $\mathrm{CW}$ refers to regions of the body that receive dual blood supply from the most distal branches of two large arteries (Figure 1E). To eliminate the effect of small infarctions, 3D BRAVO was used to assess the enlarged PVS (see Fgure S1). The severity of enlarged PVS in the CS, CW, and BG region was assessed by following a 4-point rating ordinal scale (27) (1: 1-10 enlarged PVS; 2: 11-20 enlarged PVS; 3: 21-40 enlarged PVS; 4: >40 enlarged PVS). All data were estimated by two experienced neuro-radiologists blind to the clinical data.

\section{Cerebral blood flow (CBF) calculation}

Each ASL image series was first evaluated for subject motion. Quality controls were applied to exclude unqualified results, which were defined as results from subjects whose head motion exceeded $2 \mathrm{~mm}$ of translation or $2^{\circ}$ of rotation during the ASL acquisition. An ASL difference image was calculated using a single-compartment model after subtracting the label image from the control image. The 3 ASL difference images were averaged to calculate the $\mathrm{CBF}$ maps combined with the proton densityweighted reference images (28).

Anemia, generally decreasing hemoglobin level, is common in HD patients. Relaxation time $\left(T_{1}\right)$ of blood $\left(T_{1 B}\right)$ varies with age and hematocrit or hemoglobin levels (11). CBF quantification results are significantly modified by the $\mathrm{T}_{1 \mathrm{~B}}$. To achieve rigorous CBF quantification, a singlecompartment model was used for CBF quantification (25). The hemoglobin levels of participants and the previously proposed general model were used to estimate the $\mathrm{T}_{1 \mathrm{~B}}$ in this study $(11,29)$. The hemoglobin levels of HD patients were derived from blood samples obtained during standard HD treatments; an age- and sex-dependent hemoglobin level was applied for HCs (30). $T 1_{B}$ was estimated by using a fixed normal arterial blood oxygen saturation level of 0.97 for all participants.

The data preprocessing was carried out with Statistical Parametric Mapping (SPM8). Using a nonlinear transformation in SPM8, the CBF images of the $51 \mathrm{HCs}$ were co-registered to a positron emission tomography (PET) perfusion template in the Montreal Neurological Institute (MNI) space. The mean co-registered CBF map of the $51 \mathrm{HCs}$ was defined as the standard CBF template. The CBF maps of all participants (HCs and patients) were coregistered to the standard $\mathrm{CBF}$ template of the MNI and resampled to a $2 \times 2 \times 2 \mathrm{~mm}$ voxel size. An 8 -mm full-width at half-maximum (FWHM) Gaussian kernel was used to smooth each co-registered CBF image. The CBF of each voxel was normalized by dividing by the mean $\mathrm{CBF}$ of the 

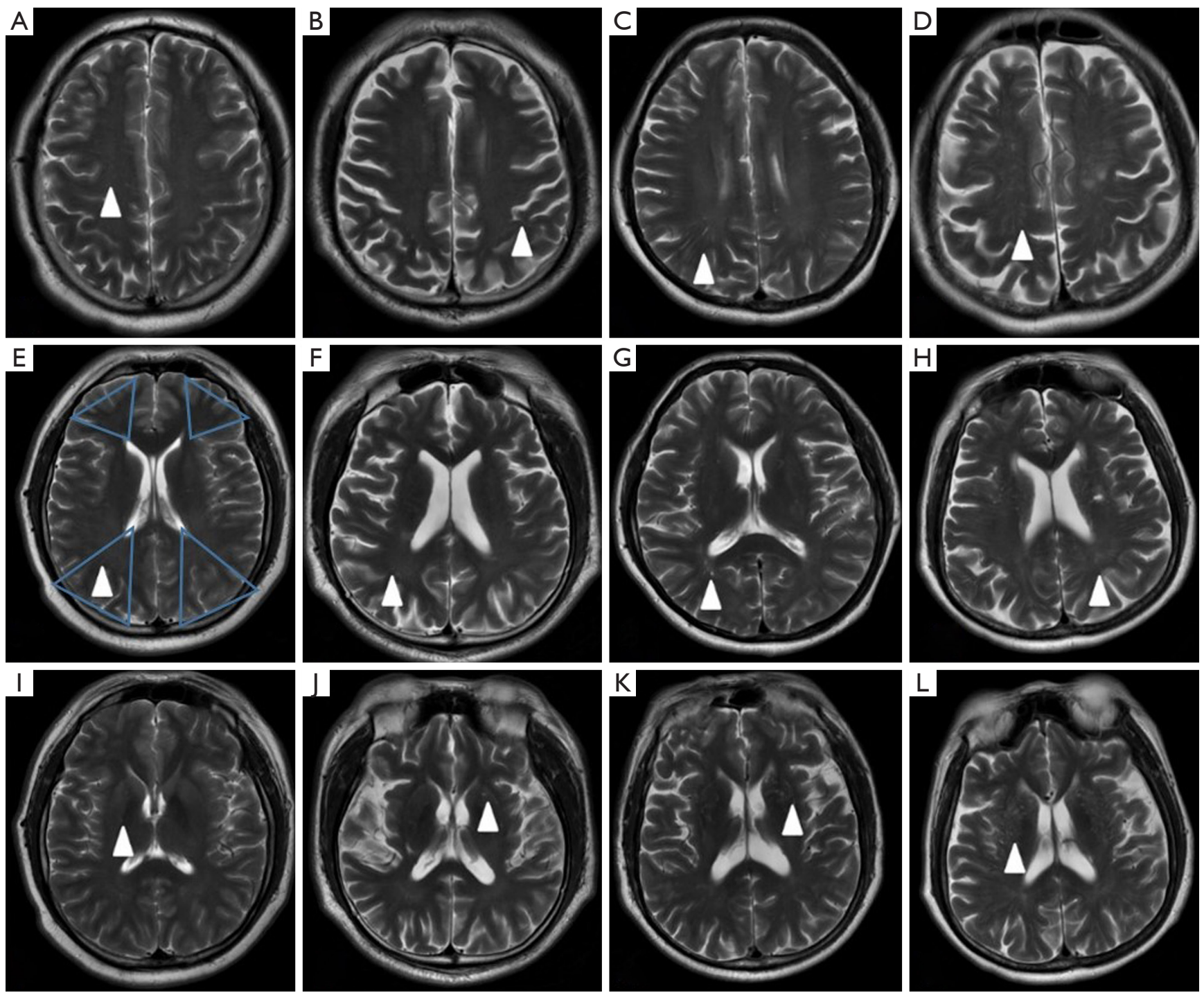

Figure 1 Examples of different categories of enlarged PVS (arrowheads) scoring from 1 to 4 at the level of the CS, CW area, and BG area. (A-D) PVS in the CS; from left to right, the scores go from 1 to 4. (E-H) PVS in the CW area (marked blue); from left to right, the scores go from 1 to 4. (I-L) PVS in the BG area; from left to right, the scores go from 1 to 4. PVS, perivascular space; CS, centrum semiovale; CW, cerebral watershed; BG, basal ganglia.

whole brain (31).

\section{White matter volume (WMV) calculation}

CAT12 (http://www.neuro.uni-jena.de/cat/) was utilized to calculate the WMV (32). Firstly, spatially co-registered T1-weighted images were normalized to the MNI space by applying the Diffeomorphic Anatomical Registration Through Exponentiated Lie Algebra (DARTEL) algorithm.
Secondly, the bias-field inhomogeneity was corrected. Thirdly, applying the standard unified segmentation model, the structural images were segmented into WM, GM, and CSF. Due to spatial registration, the segmentations were normalized by scaling with plenty of volume changes. The total amount of GM in the normalized image remained the same as in the original image. Finally, a 6-mm FWHM Gaussian kernel was used to smooth the WMV images to reduce inter-individual variability. 
Table 1 Demographic and clinical characteristics of the participants

\begin{tabular}{|c|c|c|}
\hline Characteristics & HD group $(n=51)$ & $\mathrm{HC}$ group $(\mathrm{n}=51)$ \\
\hline Age (years) & $52.4 \pm 10.3$ & $52.1 \pm 9.9$ \\
\hline Sex (male/female) & $30 / 21$ & $32 / 19$ \\
\hline Education (years) & $12.1 \pm 3.5$ & $12.3 \pm 3.2$ \\
\hline HD duration (months) & $96.1 \pm 68.8$ & NA \\
\hline \multicolumn{3}{|l|}{ CS-PVS score } \\
\hline 1 & 2 & 37 \\
\hline 2 & 11 & 13 \\
\hline 3 & 28 & 1 \\
\hline 4 & 10 & 0 \\
\hline \multicolumn{3}{|l|}{ CW-PVS score } \\
\hline 1 & 0 & 3 \\
\hline 2 & 5 & 44 \\
\hline 3 & 26 & 4 \\
\hline 4 & 20 & 0 \\
\hline \multicolumn{3}{|l|}{ BG-PVS score } \\
\hline 1 & 39 & 44 \\
\hline 2 & 9 & 5 \\
\hline 3 & 2 & 2 \\
\hline 4 & 1 & 0 \\
\hline Pre-HD SBP $(\mathrm{mmHg})$ & $143 \pm 14.3$ & \\
\hline Pre-HD DBP (mmHg) & $68 \pm 11.2$ & \\
\hline Intra-HD SBP (mmHg) & $131 \pm 13.8$ & \\
\hline Intra-HD DBP (mmHg) & $79 \pm 9.9$ & \\
\hline Post-HD SBP (mmHg) & $138 \pm 15.8$ & \\
\hline Post-HD DBP (mmHg) & $71 \pm 12.1$ & \\
\hline
\end{tabular}

Data are presented as the mean \pm standard deviation or number. HD, hemodialysis; HC, healthy control; HD duration, hemodialysis duration; CS-PVS, centrum semiovale perivascular space; BG-PVS, basal ganglia perivascular space; CW-PVS, cerebral watershed perivascular space; Pre-HD SBP, prehemodialysis systolic blood pressure; Intra-HD SBP, intrahemodialysis systolic blood pressure; Post-HD SBP, post hemodialysis systolic blood pressure; Pre-HD DBP, prehemodialysis diastolic blood pressure; Intra-HD DBP, intrahemodialysis diastolic blood pressure; Post-HD DBP, post hemodialysis diastolic blood pressure; NA, not applicable.

\section{Statistical analysis}

Statistical analysis was performed using SPSS v.19.0 (Chicago, IL). Two-tailed $t$-tests $\chi^{2}$ tests, Mann-Whitney $\mathrm{U}$ tests, and Friedman $M$ tests were used to analyze the descriptive differences, depending on the data sample. We set statistical significance at $\mathrm{P}<0.05$. Group differences in blood pressure (BP) among the three readings (pre$\mathrm{HD}$, intra-HD, and post-HD) were tested using Friedman $M$ tests. We used the intraclass correlation coefficient to estimate the agreement between the two radiologists. We conducted multiple regression analyses with data from HD patients to investigate the relationship between enlarged PVS number and HD duration. Group differences in CBF and WMV were tested using voxel-wise $t$-tests with the hemoglobin level, age, and sex as covariates (cluster size $>50$ voxels). For $t$-tests of $\mathrm{CBF}$ and $\mathrm{WMV}$, we used an FDR method with a significance threshold of $\mathrm{P}<0.05$.

\section{Results}

\section{Demographics and clinical characteristics}

Fifty-one subjects with HD and 51 healthy individuals in total were enrolled in this study. The intraclass correlation coefficient (ICC) coefficients for the CS-PVS, CWPVS, and BG-PVS were $0.815,0.823$, and 0.842 . The demographic and clinical features of the participants are shown in Table 1. The two groups were well-matched in terms of sex $\left(\chi^{2}=0.17, \mathrm{P}=0.685\right)$, education (two-sample $t$-tests, $\mathrm{t}=0.03, \mathrm{P}=0.871$ ), and age (two-sample $t$-tests, $\mathrm{t}=0.05$, $\mathrm{P}=0.819$ ). Moreover, there was no significant difference in the PVS score for the BG-PVS, and there were significant differences between the HD group and HCs group in the PVS score for the CS-PVS and CW-PVS. A Friedman M test showed differences in the systolic blood pressure (SBP) and diastolic blood pressure (DBP) of HD patients among the three readings taken (pre-HD, intra-HD, and postHD).

\section{Correlations between HD duration and PVS number in HD patients}

The CS-PVS number had a significant positive correlation 

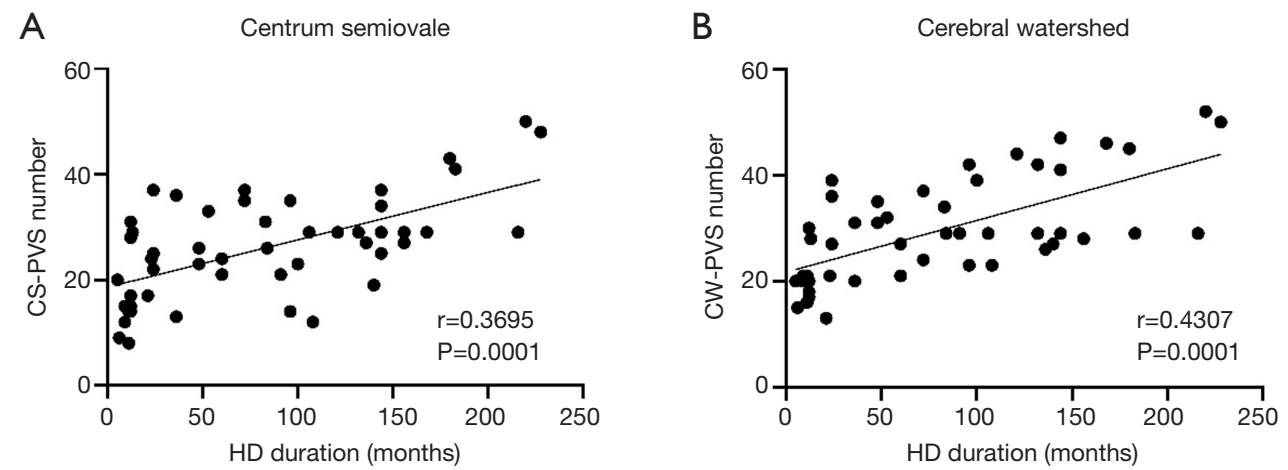

Figure 2 Correlations between HD duration and PVS number in HD patients. (A) A significant positive association was noted between HD duration and the CS-PVS number in HD patients. (B) A significant positive association was shown between HD duration and the CW-PVS number in HD patients. CS-PVS, centrum semiovale perivascular space; CW-PVS, cerebral watershed perivascular space; HD, hemodialysis; HD duration, hemodialysis duration; PVS, perivascular space.
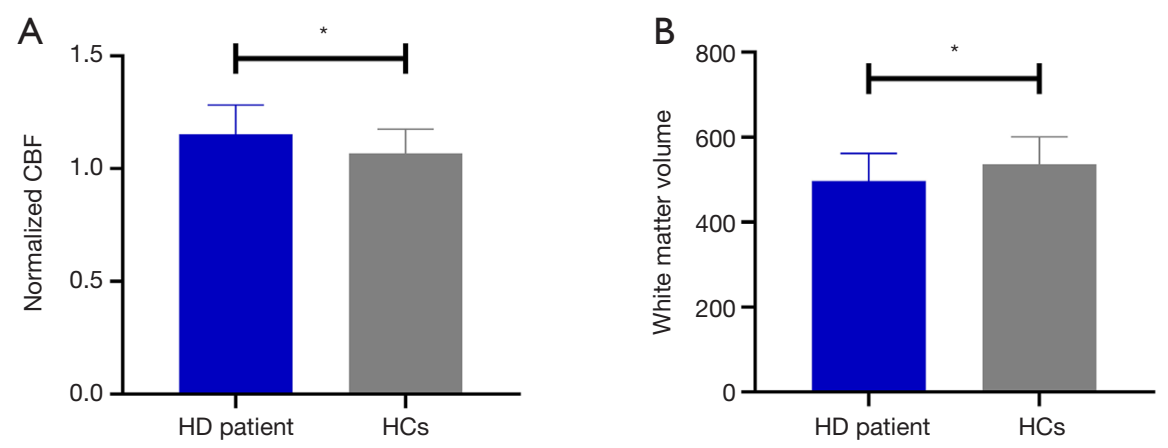

Figure 3 White matter normalized CBF and volume alterations in HD patients and HCs. (A) The HD group showed increased CBF compared to the HCs group in the WM. (B) The HD group showed decreased WMV compared to the HCs group. Covariance analysis adjusted for age and sex was conducted for the intergroup comparisons. Horizontal bars $=$ significant differences. ${ }^{*} \mathrm{P}<0.05$. $\mathrm{CBF}$, cerebral brain fluid; HCs, healthy controls; HD, hemodialysis; WM, white matter; WMV, white matter volume.

with HD duration in the HD patients $(\mathrm{r}=0.3695, \mathrm{P}=0.0001)$ (Figure $2 A$ ). The CW-PVS number had a significant positive correlation with HD duration in the HD patients $(\mathrm{r}=0.4307, \mathrm{P}=0.0001)$ (Figure 2B). We did not find significant correlations between HD duration and BG-PVS number.

\section{White matter CBF and volume alterations in HD patients and HCs}

The HD group showed increased CBF and decreased WMV in the WM compared to the HCs group (Figure 3).

\section{White matter CBF in HD patients and $\mathrm{HCs}$}

The HD group showed increased CBF in the bilateral CS,
$\mathrm{CW}$, corona radiata, and $\mathrm{BG}$ areas compared to the $\mathrm{HCs}$ group (Figure 4).

\section{White matter volume in HD patients and HCs}

The HD group showed decreased WMV in the bilateral CS, CW, corona radiata, and BG areas compared to the HCs group (Figure 5).

\section{Correlations between PVS number and white matter CBF and white matter volume in HD patients}

In the $\mathrm{CW}$ regions demonstrating $\mathrm{CBF}$ alterations, the CW-PVS number had a significant positive correlation with normalized CBF of CW in the HD patients $(r=0.2996$, 


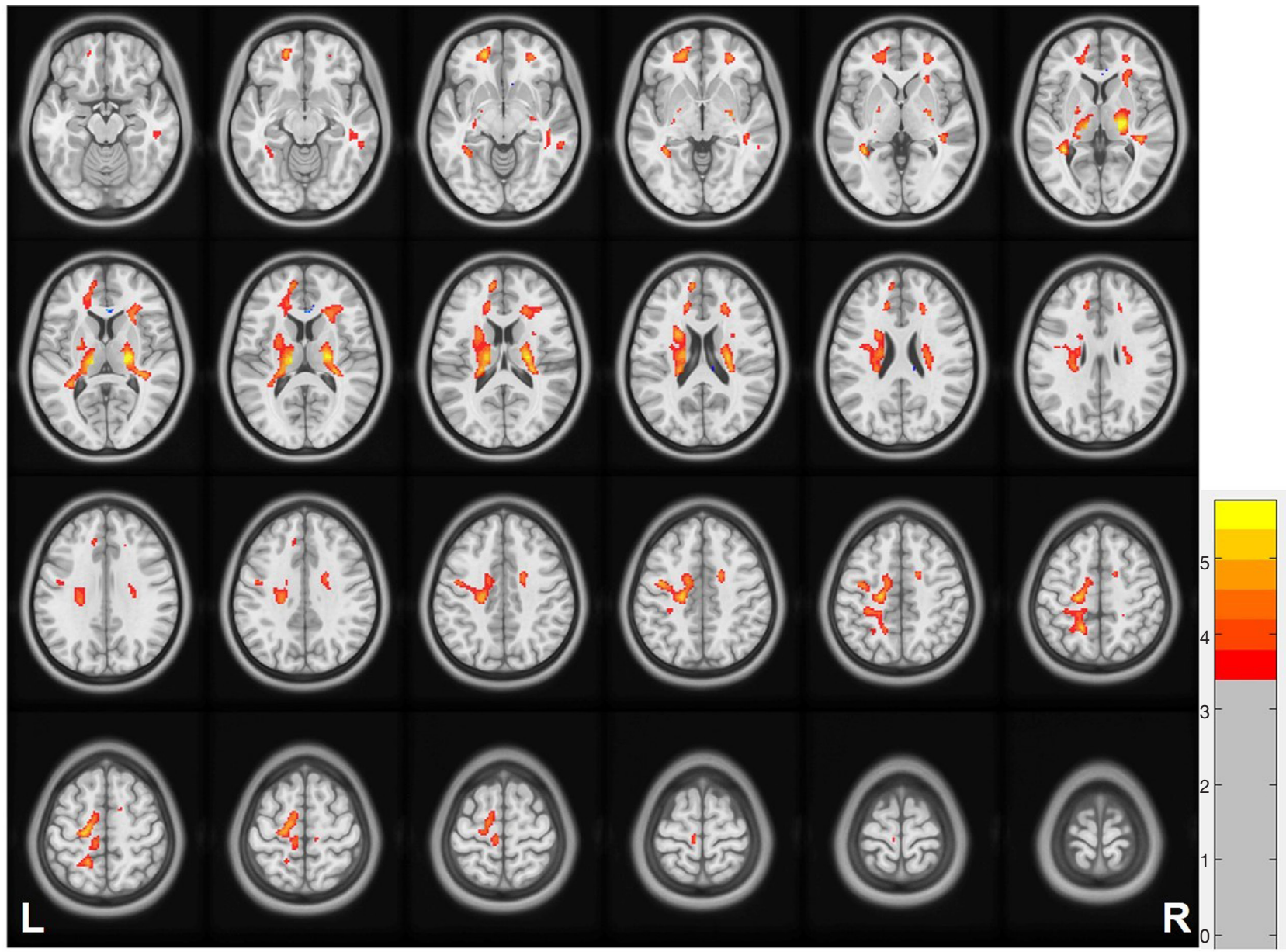

Figure 4 Brain regions exhibiting different CBF values between the HD patients and HCs. The HD group showed increased CBF compared to the HCs group in the bilateral CW areas, CS, corona radiata, and BG areas. Two-sample $t$-test; $\mathrm{P}<0.05$, with FDR correction. The CBF results with HD group > HCs group are in red. L, left; R, right. CBF, cerebral brain fluid; CS, centrum semiovale; CW, cerebral watershed; FDR, false discovery rate; HCs, healthy controls; HD, hemodialysis.

$\mathrm{P}=0.0327$ ) (Figure 6A). In the $\mathrm{CW}$ regions demonstrating WMV alterations, the CW-PVS number had a significant positive correlation with decreased $\mathrm{WMV}$ of $\mathrm{CW}$ in the HD patients $(\mathrm{r}=0.3149, \mathrm{P}=0.0244)$ (Figure $6 B)$. We did not find significant correlations between BG-PVS number and normalized $\mathrm{CBF}$ and decreased WMV.

\section{Discussion}

PVS, which hold fluid, generated waste, and compounds from the brain, surround the brain perforating vessels and constitute part of the lymphatic system. Recent studies have highlighted that enlarged PVS is likely to result in part from inflammation (33), modifications of cerebrovascular reactivity (CVR) and CBF (34,35), blood-brain barrier (BBB) abnormalities, and elevated extra-cranial and intracranial vascular pulsatility $(36,37)$. Understanding the mechanisms leading to enlarged PVS may provide valuable insight into the reasons for increased cognitive impairment prevalent in HD patients. Previous diffusionweighted imaging (DWI) and DTI studies $(38,39)$ have indicated that WM exhibited microstructural abnormalities and widespread cerebral tissue swelling in HD patients. However, the pathogenesis of enlarged PVS and visible structural dysfunction remains unclear in HD patients. To our knowledge, this is the first prospective multimodal image study to (I) investigate enlarged PVS alterations in WM regions in maintenance HD patients, (II) to perform 


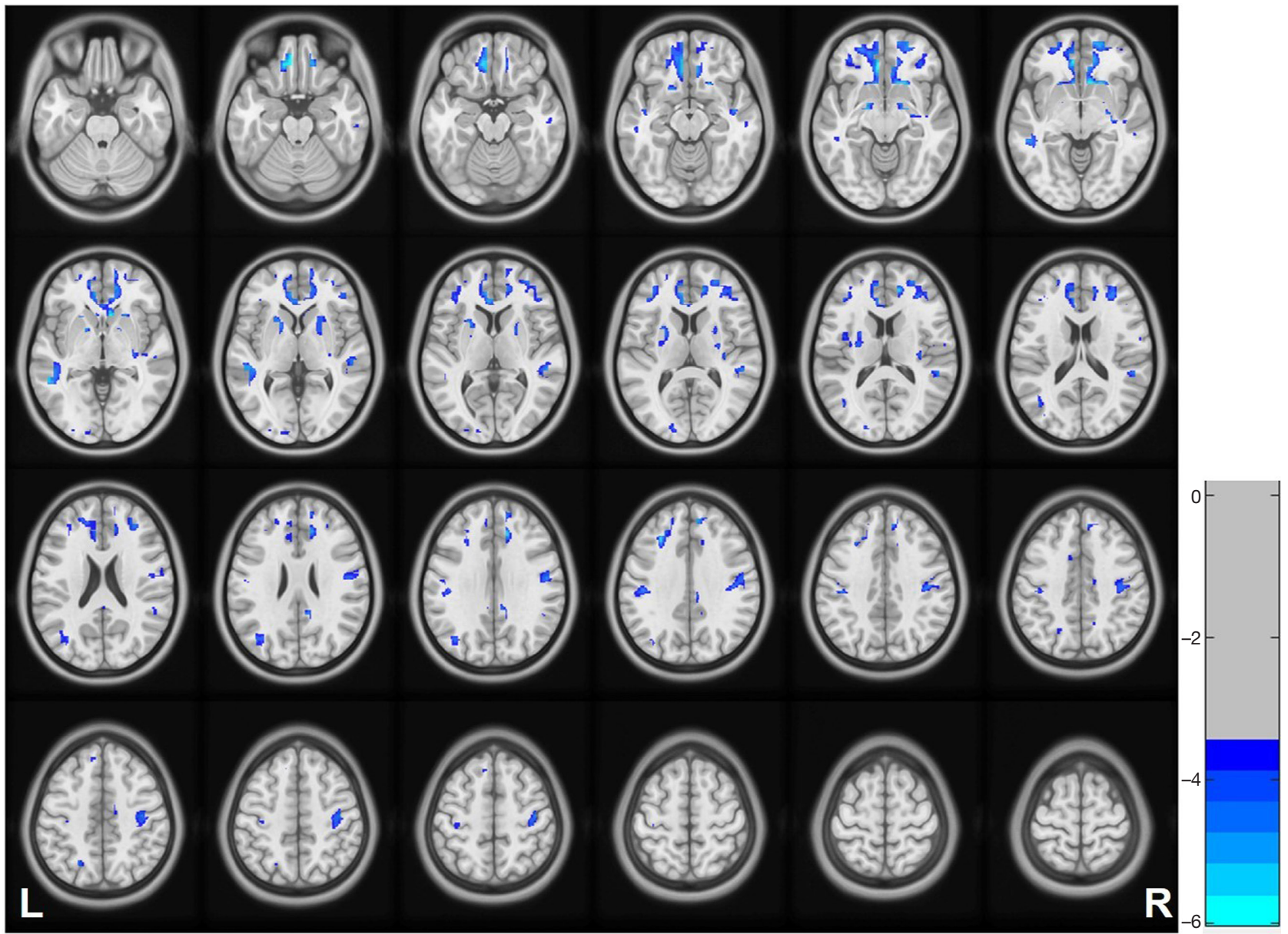

Figure 5 Brain regions WMV differences between the HD patients and the HCs group. The HD group showed decreased WMV compared to the HCs group in the bilateral $\mathrm{CW}$ areas, CS, corona radiata, and BG areas. Two-sample $t$-test; $\mathrm{P}<0.05$, with FDR correction. WMV results with $\mathrm{HD}$ group < HCs group are in blue. L, left; R, right. CS, centrum semiovale; CW, cerebral watershed; BG, basal ganglia; FDR, false discovery rate; HCs, healthy controls; HD, hemodialysis; WMV, white matter volume.

rigorous CBF quantification using individually estimated $\mathrm{T}_{1} \mathrm{~B}$ values based on hemoglobin levels to compensate for anemia effects on $T_{1} B$, and (III) to perform WMV quantification using the voxel-based analysis method. Our cross-section study results provide novel and indirect evidence supporting the association between enlarged PVS and increased $\mathrm{CBF}$ of the $\mathrm{CW}$ region and reduced WMV of the CW region in HD patients.

The observed increase in the number of enlarged PVS in HD patients is likely associated with CBF changes in the $\mathrm{CW}$ region. As a result of anemia occurring secondary to renal failure, ESRD patients show brain hyper-perfusion at rest $(40,41)$. Our results also agree with this finding. Anemia occurs early in the setting of renal disease and escalates in severity with disease progression (42). The brain vascular effects of anemia in ESRD present as reduced blood viscosity and decreased oxygen delivery. Both of these changes cause increased CBF, and several studies have reported that CBF alteration was associated with hematocrit fluctuation in ESRD (20,41). Normally, CBF is closely connected with cerebral oxygen delivery so that oxygen content in the blood is enough to meet the needs of brain metabolism. However, oxygen-carrying capacity is decreased, and brain oxygen delivery remains inadequate despite slowly increased CBF found with anemia (43). Although elevated CBF plays an important protective mechanism role by raising brain oxygenation during the course of anemia, brain oxygen delivery does not fully 

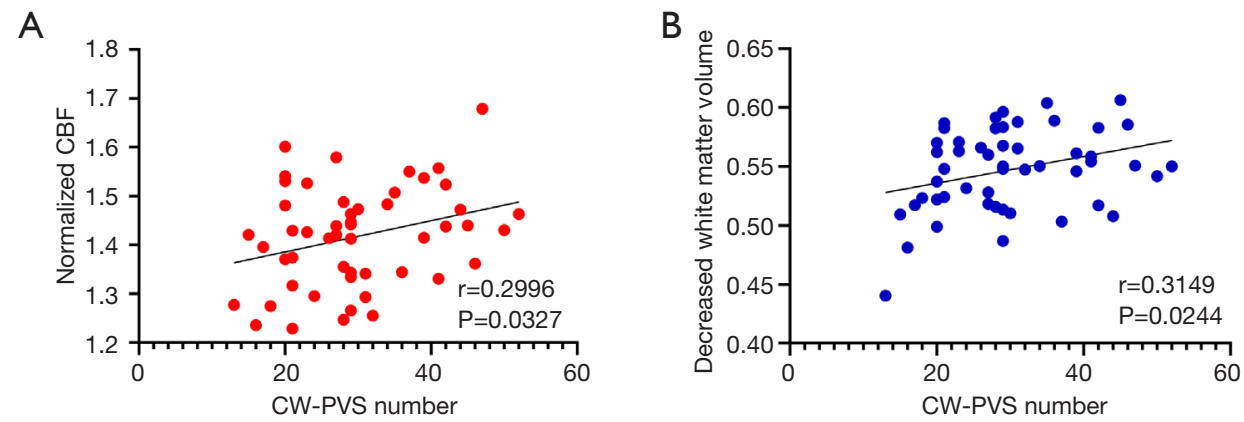

Figure 6 Correlations between PVS number and WM CBF and volume in HD patients. (A) A significant positive association was noted between CW-PVS number and the normalized CBF. (B) A significant positive association was noted between CW-PVS number and decreased WMV in HD patients. CBF, cerebral brain fluid; HD, hemodialysis; CW-PVS, cerebral watershed perivascular space; HD, hemodialysis; PVS, perivascular space; WM, white matter; WMV, white matter volume.

recover and remains damaged in ESRD at rest (43). The brain in ESRD remains over-perfused yet under oxygenated, at rest (44). Several investigations suggest that the HD process itself may exert harmful effects on the brain (45). Most studies have found that CBF decreases $7-22 \%$ during $\mathrm{HD}$ and there may be limited reserve to endure further decreases in brain oxygenation caused by decreasing CBF (46). It has been reported that repeated HD may lead to cerebral "stunning", which might be associated with cerebral perfusion change (47).

On the other hand, our study also found that the SBP and DBP during intra-HD were lower than that of the pre-HD and post-HD conditions. Due to fewer capillaries than in the cortex and the existence of CW, WM is more sensitive to perfusion than GM $(48,49)$. Therefore, we inferred that hypoxia, neuronal dying, and other neuronal pathological alterations were triggered by alteration in perfusion, which aggravates the brain environment, and the inflammation, demyelination, and dysfunction of the BBB accelerates the enlargement of PVS. In addition, because of disturbances in calcium and phosphorus metabolism, loss of pulsatility and increased stiffness caused by calcification of cerebral vasculature is common in HD patients (50). Cerebral arterial pulsatility drives fluid flow along the brain drainage pathway (51). It then follows that weakening pulsatility and elevated stiffness could reduce waste clearance $(52,53)$. Therefore, we supposed that enlargement of the PVS might be related to dysfunction in fluid clearance caused by perfusion changes.

Reduced WMV may play a role in the observed number of enlarged PVS in HD patients. The findings of our study show that enlarged PVS of the WM regions occurs more often in long-term patients with HD and that the numbers of CS-PVS and CW-PVS are positively associated with HD duration. Our findings also showed that the CWPVS number significantly correlated with decreased WMV of CW in HD patients. This suggests that the more the number of enlarged PVS, the more severe the WM atrophy. Prohovnik et al. also found that serious brain atrophy in HD patients was associated with dialysis time (54). WM alterations after HD have been reported in only a few studies utilizing conventional MRI $(55,56)$. In sum, these studies showed that persistent alteration of perfusion of the brain occurring with HD might contribute to progressive brain atrophy over time in HD patients (43). Cerebral atrophy may lead to perivascular structures widening. Maclullich et al. (57) indicated that enlarged PVS was not only a form of relatively obvious pathological alteration observed in different disease states but also a benign manifestation of aging.

Furthermore, we also revealed that CS-PVS, CWPVS, and BG-PVS were differentially affected, which was inconsistent with previous results in patients with SVD $(58,59)$. This location-specific discrepancy may suggest that the functions of CS-PVS and CW-PVS differ from those of BG-PVS. Recent studies have reported that the anatomical structures of the PVS in the BG areas differ from deep WM around the CS and CW regions (26,60). BG-PVS are branching or winding, and CS-PVS do not go through the subarachnoid space on the brain surface (61). Yamada et al. (62) highlighted that the superficial artery penetrating the brain's surface has a lower pulse pressure wave amplitude and smaller flow volume than the lenticulostriate arteriole penetrating the $\mathrm{BG}$. The $\mathrm{CS}$ and $\mathrm{CW}$ regions 
are more relatively vulnerable than the $\mathrm{BG}$ regions in $\mathrm{HD}$ patients, which was supported by our results. Therefore, the atrophy of deep WM related to long-term HD might be associated with enlarged PVS.

Our study still has several limitations that need further exploration. Firstly, as well understood, due to the low perfusion level and low signal-noise ratio (SNR) of ASL imaging, it remains challenging to measure $\mathrm{WM}$ CBF using the ASL method today. The low imaging resolution in this study and a large smoothing kernel in the normalized space will inevitably result in partial volume effects. Therefore, an alternative high-resolution imaging sequence, such as multiband EPI PCASL, should be used in the future. Secondly, further studies should investigate the relationship between cognitive impairment and enlarged PVS. Thirdly, when the FDR correction was used to analyze the relationship between WMV and HD duration, no cluster was statistically significant. A possible reason might be that the sample size of the HD patients was relatively small. Finally, the brain is subject to age-related physiological changes such as atrophy, and this should be taken into consideration when taking measurements.

In conclusion, enlarged CS-PVS and CW-PVS on MRI might be features of long-term HD in patients. Enlarged $\mathrm{CW}$-PVS are associated with higher $\mathrm{CBF}$ in the $\mathrm{CW}$ region and lower WMV in the CW region in HD patients and warrant further study given the widespread use of $\mathrm{HD}$ in the community.

\section{Acknowledgments}

Funding: This work was supported by Natural Science Foundation of China (no. 61931013) and Beijing Scholar 2015 (Zhenchang Wang).

\section{Footnote}

Conflicts of Interest: All authors have completed the ICMJE uniform disclosure form (available at https://dx.doi. org/10.21037/qims-20-1246). The authors have no conflicts of interest to declare.

Ethical Statement: The authors are accountable for all aspects of the work, including ensuring that questions related to the accuracy or integrity of any part of the work are appropriately investigated and resolved. The study was conducted in accordance with the Declaration of Helsinki (as revised in 2013). This study was approved by the Medical
Ethics Committee of Beijing Friendship Hospital, Capital Medical University. Participants provided written informed consent (version V1.1/2018-09-17) before inclusion in the study.

Open Access Statement: This is an Open Access article distributed in accordance with the Creative Commons Attribution-NonCommercial-NoDerivs 4.0 International License (CC BY-NC-ND 4.0), which permits the noncommercial replication and distribution of the article with the strict proviso that no changes or edits are made and the original work is properly cited (including links to both the formal publication through the relevant DOI and the license). See: https://creativecommons.org/licenses/by-nc-nd/4.0/.

\section{References}

1. Foley RN, Collins AJ. End-stage renal disease in the United States: an update from the United States Renal Data System. J Am Soc Nephrol 2007;18:2644-8.

2. Zhang L, Wang F, Wang L, Wang W, Liu B, Liu J, et al. Prevalence of chronic kidney disease in China: a crosssectional survey. Lancet 2012;379:815-22.

3. Arieff AI. Dialysis disequilibrium syndrome: current concepts on pathogenesis and prevention. Kidney Int 1994;45:629-35.

4. Brouns R, De Deyn PP. Neurological complications in renal failure: a review. Clin Neurol Neurosurg 2004;107:1-16.

5. Tzamaloukas AH, Agaba EI. Neurological manifestations of uraemia and chronic dialysis. Niger J Med 2004;13:98-105.

6. Pappius HM, Oh JH, Dossetor JB. The effects of rapid hemodialysis on brain tissues and cerebrospinal fluid of dogs. Can J Physiol Pharmacol 1967;45:129-47.

7. Chou MC, Hsieh TJ, Lin YL, Hsieh YT, Li WZ, Chang JM, Ko CH, Kao EF, Jaw TS, Liu GC. Widespread white matter alterations in patients with end-stage renal disease: a voxelwise diffusion tensor imaging study. AJNR Am J Neuroradiol 2013;34:1945-51.

8. Hsieh TJ, Chang JM, Chuang HY, Ko CH, Hsieh ML, Liu GC, Hsu JS. End-stage renal disease: in vivo diffusiontensor imaging of silent white matter damage. Radiology 2009;252:518-25.

9. Zhang R, Liu K, Yang L, Zhou T, Qian S, Li B, Peng Z, Li M, Sang S, Jiang Q, Sun G. Reduced white matter integrity and cognitive deficits in maintenance hemodialysis ESRD patients: a diffusion-tensor study. Eur 
Radiol 2015;25:661-8.

10. Yin Y, Li M, Li C, Ma X, Yan J, Wang T, Fu S, Hua K, Wu Y, Zhan W, Jiang G. Reduced White Matter Integrity With Cognitive Impairments in End Stage Renal Disease. Front Psychiatry 2018;9:143.

11. Li X, Slinin YX, Zhang L, Dengel DR, Tupper D, Metzger GJ, Murray AM. Cerebral blood flow characteristics following hemodialysis initiation in older adults: A prospective longitudinal pilot study using arterial spin labeling imaging. Neuroimage Clin 2020;28:102434.

12. Mu J, Ma L, Ding D, Ma X, Li P, Li R, Zhang M, Liu J. White matter characteristics between amygdala and prefrontal cortex underlie depressive tendency in end stage renal disease patients before the dialysis initiation. Brain Imaging Behav 2021;15:1815-27.

13. Richerson WT, Umfleet LG, Schmit BD, Wolfgram DF. Changes in Cerebral Volume and White Matter Integrity in Adults on Hemodialysis and Relationship to Cognitive Function. Nephron 2021;145:35-43.

14. Wardlaw JM, Smith EE, Biessels GJ, Cordonnier C, Fazekas F, Frayne R, et al. Neuroimaging standards for research into small vessel disease and its contribution to ageing and neurodegeneration. Lancet Neurol 2013;12:822-38.

15. Brown R, Benveniste H, Black SE, Charpak S, Dichgans M, Joutel A, Nedergaard M, Smith KJ, Zlokovic BV, Wardlaw JM. Understanding the role of the perivascular space in cerebral small vessel disease. Cardiovasc Res 2018;114:1462-73.

16. Heier LA, Bauer CJ, Schwartz L, Zimmerman RD, Morgello S, Deck MD. Large Virchow-Robin spaces: MR-clinical correlation. AJNR Am J Neuroradiol 1989;10:929-36.

17. Skrobot OA, Attems J, Esiri M, Hortobágyi T, Ironside JW, Kalaria RN, King A, Lammie GA, Mann D, Neal J, Ben-Shlomo Y, Kehoe PG, Love S. Vascular cognitive impairment neuropathology guidelines (VCING): the contribution of cerebrovascular pathology to cognitive impairment. Brain 2016;139:2957-69.

18. Pantoni L. Cerebral small vessel disease: from pathogenesis and clinical characteristics to therapeutic challenges. Lancet Neurol 2010;9:689-701.

19. Wuerfel J, Haertle M, Waiczies H, Tysiak E, Bechmann I, Wernecke KD, Zipp F, Paul F. Perivascular spaces-MRI marker of inflammatory activity in the brain? Brain 2008;131:2332-40.

20. Cheng BC, Chen PC, Chen PC, Lu CH, Huang YC, Chou KH, Li SH, Lin AN, Lin WC. Decreased cerebral blood flow and improved cognitive function in patients with end-stage renal disease after peritoneal dialysis: An arterial spin-labelling study. Eur Radiol 2019;29:1415-24.

21. Wang H, Han X, Jin M, Wang LY, Diao ZL, Guo W, Zhang P, Wang Z, Lv H, Ding HY, Zhang ZY, Zhao PF, Li J, Yang ZH, Liu WH, Wang ZC. Cerebral blood flow alterations in hemodialysis patients with and without restless legs syndrome: an arterial spin labeling study. Brain Imaging Behav 2021;15:401-9.

22. Wang H, Han X, Jin M, Zhang P, Wang Z, Lv H, Ding HY, Zhang ZY, Zhao PF, Li J, Yang ZH, Liu WH, Wang ZC. Patterns of Gray Matter Volume Alterations in Hemodialysis Patients With and Without Restless Legs Syndrome: Evidence From a Voxel-Based Morphometry Study. J Comput Assist Tomogr 2020;44:533-9.

23. Di Perri C, Bahri MA, Amico E, Thibaut A, Heine L, Antonopoulos G, Charland-Verville V, Wannez S, Gomez F, Hustinx R, Tshibanda L, Demertzi A, Soddu A, Laureys $\mathrm{S}$. Neural correlates of consciousness in patients who have emerged from a minimally conscious state: a crosssectional multimodal imaging study. Lancet Neurol 2016;15:830-42.

24. Mu J, Liu X, Ma S, Chen T, Ma X, Li P, Ding D, Liu J, Zhang $M$. The variation of motor-related brain structure and its relation to abnormal motor behaviors in end-stage renal disease patients with restless legs syndrome. Brain Imaging Behav 2020;14:42-50.

25. Buxton RB, Frank LR, Wong EC, Siewert B, Warach S, Edelman RR. A general kinetic model for quantitative perfusion imaging with arterial spin labeling. Magn Reson Med 1998;40:383-96.

26. Wardlaw JM, Smith C, Dichgans M. Small vessel disease: mechanisms and clinical implications. Lancet Neurol 2019;18:684-96.

27. Potter GM, Chappell FM, Morris Z, Wardlaw JM. Cerebral perivascular spaces visible on magnetic resonance imaging: development of a qualitative rating scale and its observer reliability. Cerebrovasc Dis 2015;39:224-31.

28. Xu G, Rowley HA, Wu G, Alsop DC, Shankaranarayanan A, Dowling M, Christian BT, Oakes TR, Johnson SC. Reliability and precision of pseudo-continuous arterial spin labeling perfusion MRI on 3.0 T and comparison with 15O-water PET in elderly subjects at risk for Alzheimer's disease. NMR Biomed 2010;23:286-93.

29. Hales PW, Kirkham FJ, Clark CA. A general model to calculate the spin-lattice (T1) relaxation time of blood, accounting for haematocrit, oxygen saturation and magnetic field strength. J Cereb Blood Flow Metab 
2016;36:370-4.

30. Mahlknecht U, Kaiser S. Age-related changes in peripheral blood counts in humans. Exp Ther Med 2010;1:1019-25.

31. Aslan S, Lu H. On the sensitivity of ASL MRI in detecting regional differences in cerebral blood flow. Magn Reson Imaging 2010;28:928-35.

32. Liu F, Tian H, Li J, Li S, Zhuo C. Altered voxel-wise gray matter structural brain networks in schizophrenia: Association with brain genetic expression pattern. Brain Imaging Behav 2019;13:493-502.

33. Vos CM, Geurts JJ, Montagne L, van Haastert ES, Bö L, van der Valk P, Barkhof F, de Vries HE. Blood-brain barrier alterations in both focal and diffuse abnormalities on postmortem MRI in multiple sclerosis. Neurobiol Dis 2005;20:953-60.

34. Joutel A, Monet-Leprêtre M, Gosele C, Baron-Menguy C, Hammes A, Schmidt S, Lemaire-Carrette B, Domenga V, Schedl A, Lacombe P, Hubner N. Cerebrovascular dysfunction and microcirculation rarefaction precede white matter lesions in a mouse genetic model of cerebral ischemic small vessel disease. J Clin Invest 2010;120:433-45.

35. Lacombe P, Oligo C, Domenga V, Tournier-Lasserve E, Joutel A. Impaired cerebral vasoreactivity in a transgenic mouse model of cerebral autosomal dominant arteriopathy with subcortical infarcts and leukoencephalopathy arteriopathy. Stroke 2005;36:1053-8.

36. Riba-Llena I, Jiménez-Balado J, Castañé X, Girona A, López-Rueda A, Mundet X, Jarca CI, ÁlvarezSabin J, Montaner J, Delgado P. Arterial Stiffness Is Associated With Basal Ganglia Enlarged Perivascular Spaces and Cerebral Small Vessel Disease Load. Stroke 2018;49:1279-81.

37. Shi Y, Thrippleton MJ, Blair GW, Dickie DA, Marshall I, Hamilton I, Doubal FN, Chappell F, Wardlaw JM. Small vessel disease is associated with altered cerebrovascular pulsatility but not resting cerebral blood flow. J Cereb Blood Flow Metab 2020;40:85-99.

38. Chou MC, Ko CH, Hsieh TJ, Chang JM, Chung WS. A preliminary report of longitudinal white matter alterations in patients with end-stage renal disease: A three-year diffusion tensor imaging study. PLoS One 2019;14:e0215942.

39. Schaier M, Wolf RC, Kubera K, Nagel S, Bartsch A, Zeier M, Bendszus M, Herweh C. Vasogenic Brain Edema During Maintenance Hemodialysis : Preliminary Results from Tract-based Spatial Statistics and Voxel-based Morphometry. Clin Neuroradiol 2021;31:217-24. 40. Jiang XL, Wen JQ, Zhang LJ, Zheng G, Li X, Zhang Z,
Liu Y, Zheng LJ, Wu L, Chen HJ, Kong X, Luo S, Lu GM, Ji XM, Zhang ZJ. Cerebral blood flow changes in hemodialysis and peritoneal dialysis patients: an arterial-spin labeling MR imaging. Metab Brain Dis 2016;31:929-36.

41. Zheng G, Wen J, Yu W, Li X, Zhang Z, Chen H, Kong X, Luo S, Jiang X, Liu Y, Zhang Z, Zhang LJ, Lu GM. Anemia rather than hypertension contributes to cerebral hyperperfusion in young adults undergoing hemodialysis: A phase contrast MRI study. Sci Rep 2016;6:22346.

42. St Peter WL, Guo H, Kabadi S, Gilbertson DT, Peng Y, Pendergraft T, Li S. Prevalence, treatment patterns, and healthcare resource utilization in Medicare and commercially insured non-dialysis-dependent chronic kidney disease patients with and without anemia in the United States. BMC Nephrol 2018;19:67.

43. Sprick JD, Nocera JR, Hajjar I, O'Neill WC, Bailey J, Park J. Cerebral blood flow regulation in end-stage kidney disease. Am J Physiol Renal Physiol 2020;319:F782-91.

44. Matsukawa S, Hamada M, Mizota T. Low preoperative regional cerebral oxygen saturation in hemodialysis patients. JA Clin Rep 2017;3:13.

45. Skinner H, Mackaness C, Bedforth N, Mahajan R. Cerebral haemodynamics in patients with chronic renal failure: effects of haemodialysis. Br J Anaesth 2005;94:203-5.

46. Stefanidis I, Bach R, Mertens PR, Liakopoulos V, Liapi G, Mann H, Heintz B. Influence of hemodialysis on the mean blood flow velocity in the middle cerebral artery. Clin Nephrol 2005;64:129-37.

47. Findlay MD, Dawson J, Dickie DA, Forbes KP, McGlynn D, Quinn T, Mark PB. Investigating the Relationship between Cerebral Blood Flow and Cognitive Function in Hemodialysis Patients. J Am Soc Nephrol 2019;30:147-58.

48. Lin J, Wang D, Lan L, Fan Y. Multiple Factors Involved in the Pathogenesis of White Matter Lesions. Biomed Res Int 2017;2017:9372050.

49. Varga AW, Johnson G, Babb JS, Herbert J, Grossman RI, Inglese $M$. White matter hemodynamic abnormalities precede sub-cortical gray matter changes in multiple sclerosis. J Neurol Sci 2009;282:28-33.

50. Moon H, Chin HJ, Na KY, Joo KW, Kim YS, Kim S, Han SS. Hyperphosphatemia and risks of acute kidney injury, end-stage renal disease, and mortality in hospitalized patients. BMC Nephrol 2019;20:362.

51. Iliff JJ, Wang M, Zeppenfeld DM, Venkataraman A, Plog BA, Liao Y, Deane R, Nedergaard M. Cerebral arterial pulsation drives paravascular CSF-interstitial fluid exchange in the murine brain. J Neurosci 
2013;33:18190-9.

52. Bedussi B, van Lier MG, Bartstra JW, de Vos J, Siebes M, VanBavel E, Bakker EN. Clearance from the mouse brain by convection of interstitial fluid towards the ventricular system. Fluids Barriers CNS 2015;12:23.

53. Tarasoff-Conway JM, Carare RO, Osorio RS, Glodzik L, Butler T, Fieremans E, Axel L, Rusinek H, Nicholson C, Zlokovic BV, Frangione B, Blennow K, Ménard J, Zetterberg H, Wisniewski T, de Leon MJ. Clearance systems in the brain-implications for Alzheimer disease. Nat Rev Neurol 2015;11:457-70.

54. Prohovnik I, Post J, Uribarri J, Lee H, Sandu O, Langhoff E. Cerebrovascular effects of hemodialysis in chronic kidney disease. J Cereb Blood Flow Metab 2007;27:1861-9.

55. Ağildere AM, Benli S, Erten Y, Coşkun M, Boyvat F, Ozdemir N. Osmotic demyelination syndrome with a dysequilibrium syndrome: reversible MRI findings. Neuroradiology 1998;40:228-32.

56. Lakadamyali H, Ergün T. MRI for acute neurologic complications in end-stage renal disease patients on hemodialysis. Diagn Interv Radiol 2011;17:112-7.

57. Maclullich AM, Wardlaw JM, Ferguson KJ, Starr JM, Seckl JR, Deary IJ. Enlarged perivascular spaces are

Cite this article as: Wang $\mathrm{H}$, Han $\mathrm{X}$, Li M, Yang $\mathrm{ZH}$, Liu WH, Wang ZC. Long-term hemodialysis may affect enlarged perivascular spaces in maintenance hemodialysis patients: evidence from a pilot MRI study. Quant Imaging Med Surg 2022;12(1):341-353. doi: 10.21037/qims-20-1246 associated with cognitive function in healthy elderly men. J Neurol Neurosurg Psychiatry 2004;75:1519-23.

58. Charidimou A, Hong Y'T, Jäger HR, Fox Z, Aigbirhio FI, Fryer TD, Menon DK, Warburton EA, Werring DJ, Baron JC. White matter perivascular spaces on magnetic resonance imaging: marker of cerebrovascular amyloid burden? Stroke 2015;46:1707-9.

59. Loos CM, Klarenbeek P, van Oostenbrugge RJ, Staals J. Association between Perivascular Spaces and Progression of White Matter Hyperintensities in Lacunar Stroke Patients. PLoS One 2015;10:e0137323.

60. Zhang ET, Inman CB, Weller RO. Interrelationships of the pia mater and the perivascular (Virchow-Robin) spaces in the human cerebrum. J Anat 1990;170:111-23.

61. Ishikawa M, Yamada S, Yamamoto K. Three-dimensional observation of Virchow-Robin spaces in the basal ganglia and white matter and their relevance to idiopathic normal pressure hydrocephalus. Fluids Barriers CNS 2015;12:15.

62. Yamada S, Ishikawa M, Yamamoto K, Yamaguchi $M$, Oshima M. Location-specific characteristics of perivascular spaces as the brain's interstitial fluid drainage system. J Neurol Sci 2019;398:9-15. 

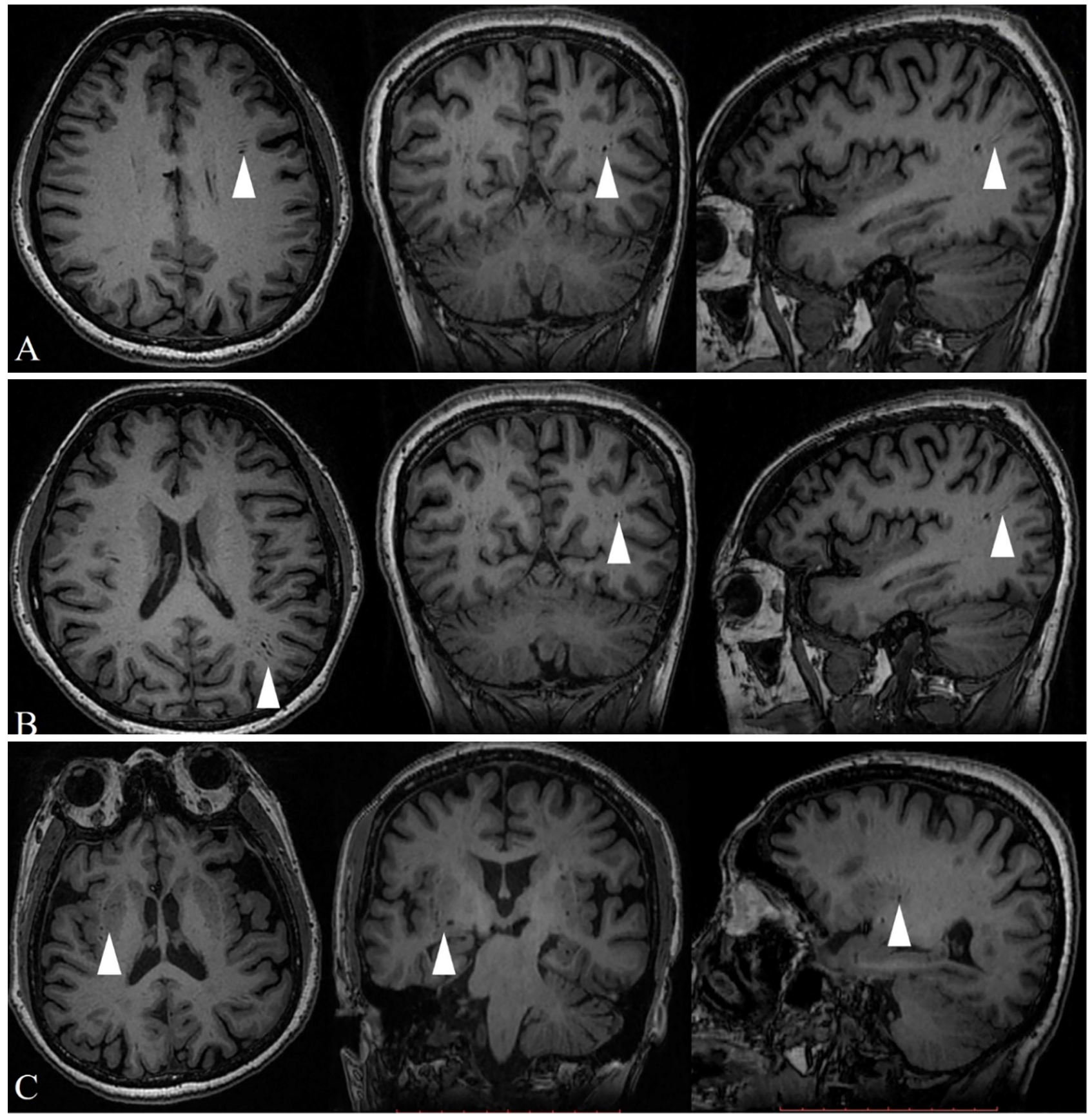

Figure S1 Supplementary examples showing different categories of 3D observations for enlarged PVS (arrowheads) at the level of the centrum semiovale (A), watershed area (B), and basal ganglia area (C). BG, basal ganglia; CS, centrum semiovale; CW, cerebral watershed; PVS, perivascular space. 